\title{
Co-Infection by Two Criniviruses Alters Accumulation of Each Virus in a Host-Specific Manner and Influences Efficiency of Virus Transmission
}

\author{
William M. Wintermantel, Arturo A. Cortez, Amy G. Anchieta, Anju Gulati-Sakhuja, and Laura L. Hladky
}

United States Department of Agriculture-Agricultural Research Service, Salinas, CA.

Accepted for publication 6 September 2008.

\begin{abstract}
Wintermantel, W. M., Cortez, A. A., Anchieta, A. G., Gulati-Sakhuja, A., and Hladky, L. L. 2008. Co-infection by two criniviruses alters accumulation of each virus in a host-specific manner and influences efficiency of virus transmission. Phytopathology 98:1340-1345.

Tomato chlorosis virus (ToCV), and Tomato infectious chlorosis virus (TICV), family Closteroviridae, genus Crinivirus, cause interveinal chlorosis, leaf brittleness, and limited necrotic flecking or bronzing on tomato leaves. Both viruses cause a decline in plant vigor and reduce fruit yield, and are emerging as serious production problems for field and greenhouse tomato growers in many parts of the world. The viruses have been found together in tomato, indicating that infection by one Crinivirus sp. does not prevent infection by a second. Transmission efficiency and virus persistence in the vector varies significantly among the four different whitefly vectors of ToCV; Bemisia tabaci biotypes A and B, Trialeurodes abutilonea, and T. vaporariorum. Only T. vaporariorum can transmit TICV. In order to elucidate the effects of co-infection on Crinivirus sp. accumulation and transmission efficiency, we established

Physalis wrightii and Nicotiana benthamiana source plants, containing either TICV or ToCV alone or both viruses together. Vectors were allowed to feed separately on all virus sources, as well as virus-free plants, then were transferred to young plants of both host species. Plants were tested by quantitative reverse-transcription polymerase chain reaction, and results indicated host-specific differences in accumulation by TICV and ToCV and alteration of accumulation patterns during coinfection compared with single infection. In $N$. benthamiana, TICV titers increased during co-infection compared with levels in single infection, while ToCV titers decreased. However, in $P$. wrightii, titers of both TICV and $\mathrm{ToCV}$ decreased during mixed infection compared with single infection, although to different degrees. Vector transmission efficiency of both viruses corresponded with virus concentration in the host in both single and mixed infections. This illustrates that Crinivirus epidemiology is impacted not only by vector transmission specificity and incidence of hosts but also by interactions between viruses and efficiency of accumulation in host plants.
\end{abstract}

In the mid-1990s, two new criniviruses emerged as threats to tomato production in North America. Tomato infectious chlorosis virus (TICV) and Tomato chlorosis virus (ToCV) cause identical symptoms on tomato, including interveinal yellowing and thickening of leaves $(7,27,28)$. Although no obvious symptoms occur on fruit, production is affected through decreased fruit size and number as well as early senescence. Both viruses have now been found in many areas throughout the world in both field and greenhouse environments. TICV is abundant in tomato-production fields along the west coast of North America in both Mexico and California (20). ToCV is common in the southeastern United States and also has been found in Puerto Rico (22), Europe $(3,5,6,8,11,12)$, Morocco (9), Taiwan (16), and the Middle East $(2,15)$.

Symptoms resulting from either TICV or ToCV infection often do not develop until 3 to 4 weeks after inoculation. If nursery plants are exposed to vector populations at an early age, it is possible for these viruses to be spread to new areas through movement of transplants prior to symptom development. Weed hosts near production areas represent another potential virus reservoir $(7,8)$. Similarly, some ornamentals can serve as reservoirs for virus infection $(7,23,26)$. Reservoir hosts near field or greenhouse

Corresponding author: W. M. Wintermantel

E-mail address: bill.wintermantel@ars.usda.gov

doi:10.1094/PHYTO-98-12-1340

This article is in the public domain and not copyrightable. It may be freely reprinted with customary crediting of the source. The American Phytopathological Society, 2008. production areas may serve as sources for whitefly feeding, resulting in movement of the viruses into surrounding fields.

Both viruses are transmitted by the greenhouse whitefly (Trialeurodes vaporariorum Westwood). However, ToCV is unique among Crinivirus spp. in that it is also transmitted by the banded wing whitefly ( $T$. abutilonea Haldeman) and Bemisia tabaci Gennadius biotypes A and B, the only Crinivirus sp. known to be transmitted by species within both genera $(23,25,26)$. Although this has been known since the initial characterization of ToCV, the efficiency of transmission by the different vector species was only recently demonstrated (23). ToCV can be transmitted equally well and with high efficiency by both $B$. tabaci biotype B and T. abulilonea using individual whiteflies of either vector. Transmission by other members of these genera was much less efficient. Single whitefly transmission was not observed either with $B$. tabaci biotype A or T. vaporariorum (23), illustrating that the relationship between the vector and the virus can significantly impact transmission efficiency. A comparison of transmission efficiency of ToCV by $T$. vaporariorum (23) with efficiency of transmission of TICV (7) indicates that TICV is much more efficiently transmitted by $T$. vaporariorum than ToCV.

A comparison of ToCV persistence in the two more efficient vectors, B. tabaci biotype B and T. abulilonea, further illustrates that each vector shares a unique relationship with the virus, and it cannot be assumed that studies conducted on one vector can be extended to others even for the same virus (23). Although transmission efficiency with $B$. tabaci biotype $\mathrm{B}$ and $T$. abulilonea were comparable between the two species, persistence of ToCV in T. abutilonea far exceeded that in B. tabaci biotype B (23). Clearly, both of these more efficient vectors retained virus in a 
transmissible form longer than either of the less efficient vectors (23). ToCV is transmitted in a semipersistent manner by its whitefly vectors, and a specific association between the virus and factors associated with each vector is likely required for transmission. One possible explanation is that this association may be more efficient with $T$. abutilonea than it is with $B$. tabaci biotype $\mathrm{B}$, resulting in better virus stability reflected by longer persistence, although this remains to be tested scientifically.

To more fully understand the relationship between virus concentration and whitefly transmission, as well as how interactions between viruses co-infecting common host plants may influence virus emergence and dominance in an infected plant, we directly compared transmission efficiency of two criniviruses, TICV and ToCV, with virus concentration in two solanaceous hosts during single and mixed infections. Results illustrate that not only does the host influence virus accumulation, but also competition by a co-infecting virus can change accumulation patterns within the host and influence whitefly transmission efficiency.

\section{MATERIALS AND METHODS}

Virus isolates. The ToCV isolate used in these studies was originally obtained from a commercial tomato-producing greenhouse in Suwannee County, FL during January 1996, and is known as the Florida isolate (ToCV-FL) $(23,24,27)$. This typeisolate of ToCV has been maintained in tomato (Solanum lycopersicum L.), Physalis wrightii Gray, and Nicotiana benthamiana Domin plants, and transferred from plant to plant via whitefly vectors, primarily B. tabaci biotype B and T. abutilonea, but also periodically with $B$. tabaci biotype A and $T$. vaporariorum to reduce selection pressure favoring isolates transmitted by specific vectors. The TICV isolate used in these studies was originally isolated from a tomato field in Orange County, CA in 1994 (7). This isolate has been maintained in tomato, P. wrightii, and N. benthamiana plants, and transferred from plant to plant via $T$. vaporariorum. To confirm pure infection of ToCV or TICV prior to inoculations, all virus source plants were tested initially by either nucleic acid spot hybridization with RNA probes specific to either TICV or ToCV (10) or reverse-transcription polymerase chain reaction (RT-PCR) using primers that amplify either the complete 773-nucleotide (nt) ToCV coat protein gene (15) or a 325-nt section of the TICV HSP70h gene (23).

Vectors. All whitefly colonies were maintained virus-free in insect-proof cages in isolated growth rooms at temperatures ranging from 26 to $32^{\circ} \mathrm{C}$ on the hosts described below. A colony of $T$. vaporariorum, originally collected in a greenhouse at the United States Department of Agriculture-Agricultural Research Service in Salinas, CA in the early $1960 \mathrm{~s}$, was reared on P. wrightii. T. abutilonea, originally collected in Illinois in the late 1970s, was reared on $P$. wrightii, Malva parviflora L., and cotton (Gossypium hirsutum L.). B. tabaci biotype B, originally collected in 1990 from melon (Cucumis melo L.) in the Imperial Valley of California, was reared on broccoli (Brassica oleracea $\mathrm{L}$.).

Virus transmission. Source plants for transmission studies were $P$. wrightii or $N$. benthamiana, inoculated approximately 30 days earlier and expressing interveinal yellowing symptoms, a characteristic of Crinivirus infection. Prior to transmission, infections were confirmed by either RT-PCR or Northern hybridization. Large populations of each whitefly vector were allowed to mass feed on virus-infected source plants for 48-h acquisition access periods (AAPs). Following virus acquisition, whiteflies were transferred to leaf cages by briefly chilling whiteflies at $4^{\circ} \mathrm{C}$ to immobilize them. Clip cages containing 30 whiteflies each were used on each plant for transmission experiments by attaching the cage to the underside of the leaf for $48 \mathrm{~h}$. Following inoculation, cages were carefully removed from leaves and remaining whiteflies were manually killed. Any residual whiteflies were killed by subsequent treatment with Resmethrin (Whitmire
Micro-Gen, St. Louis). One week post inoculation, inoculated leaves were removed from plants to prevent whitefly nymphs from maturing to adults. Plants were grown in whitefly-proof screened cages at $28^{\circ} \mathrm{C}$ with 14 -h day length.

Transmission of each virus for the establishment of single and mixed infections was performed as illustrated in Figure 1. TICV and ToCV were transmitted from original source plants using $T$. vaporariorum and T. abutilonea, respectively. Each virus was transmitted to healthy $P$. wrightii and $N$. benthamiana seedlings at the four-true-leaf stage to establish single infections containing each virus in each host. Co-infections were also established by feeding viruliferous $T$. vaporariorum and $T$. abutilonea containing TICV and ToCV, respectively, on the same test plant. Single and co-infections were confirmed using nucleic acid hybridization and RT-PCR $(10,15,23)$. Plants confirmed as positive for both viruses were analyzed by quantitative RT-PCR (qRT-PCR) as described below. It was difficult to consistently obtain co-infected plants with comparable levels of both viruses in either host, based on either hybridization or qRT-PCR. Consequently, co-infected plants with significant accumulation of both viruses were selected as source plants for downstream transmissions. Secondary transmissions were performed from the singly and doubly infected plants at 3 to 4 weeks postinoculation using both vectors for both viruses. Bouquets of tissue from each host containing single infections of each virus or double infections containing both TICV and ToCV were placed in $125-\mathrm{ml}$ flasks in separate transmission cages. T. vaporariorum and T. abutilonea were fed separately on bouquets of tissue from these sources for 48-h AAPs, then transferred to new hosts for a 48-h inoculation access period (IAP) (Fig. 1). In all experiments, virus-free whiteflies were also fed on healthy seedlings to confirm the virus-free nature of the whitefly colonies. Four weeks postinoculation, plants were analyzed for the presence and concentration of TICV and ToCV as described below.

Virus infection and concentration in plants. RNA was extracted using the RNeasy Kit (Qiagen, Valencia, CA) followed by treatment with Turbo DNAse (Ambion, Austin, TX) to eliminate residual DNA. Single and mixed infections of TICV and ToCV were confirmed by nucleic acid hybridization using RNA probes specific to each virus as described by Li et al. (10), with secondary confirmation using RT-PCR as described by Wintermantel and Wisler (23). RNA concentrations were determined using a Nanodrop spectrophotometer (Nanodrop Technologies, Wilmington, DE). Virus-specific primers were designed to sequences within the gene encoding the HSP70h protein for each virus. For TICV, primers TicQ 467F 5' TCAATTCTTTATC-

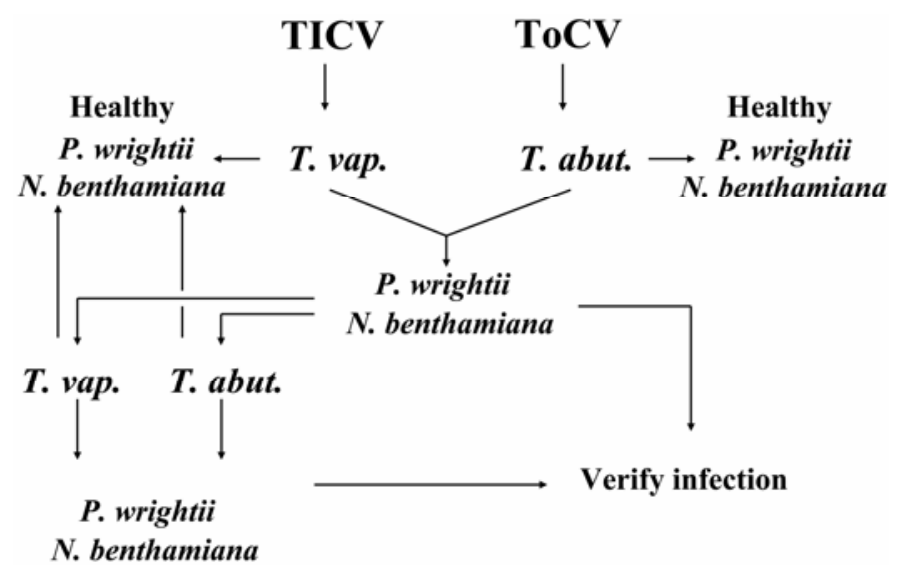

Fig. 1. Schematic diagram of transmission strategy used for development of single and mixed infections of Tomato infectious chlorosis virus (TICV) and Tomato chlorosis virus (ToCV) in two hosts, Nicotiana benthamiana and Physalis wrightii, using greenhouse whitefly (Trialeurodes vaporariorum; abbrev. T. vap.) and banded-wing whitefly (T. abutilonea; abbrev. T. abut.). 
GTTTCCTTGCAG 3' and TicQ 593R 5' TCAAATGTACCTCCACCAAAGTC 3' formed a 126-bp amplicon. For ToCV, primers TocQ 875F 5' AACCATTCGCTAGACGCAGTT $3^{\prime}$ and TocQ 998R 5' TCTGCTCTATTCTGAATCGGTCTAA 3' formed a 123bp amplicon. These primers were thoroughly tested for primerdimers, amplification of plant RNA, and cross-amplification of TICV and ToCV by melt-curve analysis. Target amplification was confirmed by cloning and sequencing of the qRT-PCR reaction products. RT was performed by heating $1.0 \mu \mathrm{g}$ of total RNA with $0.5 \mu \mathrm{M}$ virus-specific reverse primer to $95^{\circ} \mathrm{C}$ for $5 \mathrm{~min}$. RT components were added to active concentrations of 10 to $20 \mathrm{U}$ of RNasin (Promega Corp., Madison, WI), MMLV RT buffer, $1 \mathrm{mM}$ dNTPs, $5 \mathrm{mM}$ dithiothreitol, and $200 \mathrm{U}$ of MMLV Reverse Transcriptase (Promega Corp.). RT proceeded at $42^{\circ} \mathrm{C}$ for $1 \mathrm{~h}$ in a thermal cycler. qPCR was carried out on a Bio-Rad iCycler IQ with the following profile: $95^{\circ} \mathrm{C}$ for $30 \mathrm{~s}$ for three cycles to calibrate background, $95^{\circ} \mathrm{C}$ for $10 \mathrm{~min}$ to begin amplification, followed by 40 cycles of $95^{\circ} \mathrm{C}$ for $15 \mathrm{~s}$ and $60^{\circ} \mathrm{C}$ for $30 \mathrm{~s}$. qPCR reactions ( $25 \mu \mathrm{l}$ volume) included $2 \mu \mathrm{l}$ of the RT reactions, $12.5 \mu \mathrm{l}$ of Power SYBR Green Master Mix (ABI), $0.125 \mu \mathrm{M}$ forward and reverse primer, and $25 \mathrm{nmol}$ fluorescein. cDNA synthesized from healthy $N$. benthamiana was used as a negative control. Other control reactions included water blank and RNA (no cDNA template control). Each qPCR reaction was run in triplicate, with 8 to 12 replications per treatment. For standard curves, cDNA was synthesized by RT followed by qPCR, as stated above. The respective qPCR amplicons were cloned into pGEM-T Easy (Promega Corp.) using the manufacturer's protocol and sequenced on both strands using the respective primers. The plasmids were extracted from the selected colonies using Qiagen Plasmid Miniprep kit and linearized by digesting with SalI. The gel-extracted fragments were quantified on Nanodrop and used to create stable standard curves (14). The dilution series was performed by copy number following methods recommended by Applied Biosystems (Foster City, CA) (1). The cycle threshold and copy number were determined using Bio-Rad iCycler iQ Real-Time Detection System Software (version 3.0; Bio-Rad, Hercules, CA). Amplification was followed by melt-curve analysis. Statistical analysis of the data was performed on JMP 7.0 software (SAS Institute, Cary, NC).

\section{RESULTS}

Is host-specific accumulation of criniviruses altered during mixed infections? An experiment was conducted to examine competitiveness of ToCV and TICV in different hosts, and how host-specific accumulation influences vector transmission. These viruses share numerous hosts, although there are differences as well $(7,23)$. Research focused on two hosts common to a broad array of Crinivirus spp., P. wrightii and $N$. benthamiana. Plants of each host were inoculated with TICV and ToCV individually by viruliferous $T$. vaporariorum (for TICV) and T. abutilonea (for ToCV). T. abutilonea is the most efficient vector of ToCV (23) and T. vaporariorum is the only known vector of TICV (7). In addition, plants of each host were established with mixed infections of both TICV and ToCV (Fig. 1). Infections of host plants were confirmed by hybridization using molecular probes for detection of each virus or RT-PCR to identify plants with strong accumulation of each virus in single and mixed infections for use in transmission studies. Separate individual leaves from the selected plants were used for virus acquisition by both $T$. abutilonea and T. vaporariorum. Following 48-h virus acquisition periods, whiteflies were transferred to leaf cages and placed on new plants of the same species from which they originated for 48 $\mathrm{h}$, after which cages were removed and whiteflies killed with insecticide (Resmethrin; Whitmire Micro-Gen). Four weeks post transmission, virus titer was again checked using qRT-PCR to determine the level of virus accumulation in single and mixed infections in each host. Results demonstrated that each host differed in its ability to accumulate each virus and that, when both viruses co-infected a host plant, there were changes in accumulation compared with single infection, suggesting differences in compatibility or efficiency of interactions between each virus and each host.

During single infections, TICV and ToCV accumulated to comparable levels in the common host $P$. wrightii (Fig. 2). During coinfection, however, ToCV was clearly the dominant virus, accumulating to levels significantly higher than TICV $(P=$ $0.0002)$. Both viruses accumulated less during mixed infections than in single infections $(P<0.0001)$ (Fig. 2). This may be a result of competition for host factors because the two viruses are members of the same genus and may use similar host factors during infection. Results in N. benthamiana differed from those in $P$. wrightii. There was significantly higher accumulation of ToCV compared with TICV during single infections of $N$. benthamiana by each virus $(P=0.0053)$ (Fig. 3$)$. During mixed infections, however, accumulation of each virus was almost the inverse of that found in single infections in $N$. benthamiana, with TICV accumulating to higher levels than ToCV, although the differences were not significant at the $95 \%$ confidence level $(P=0.1703)$
Virus Accumulation in Physalis wrightii

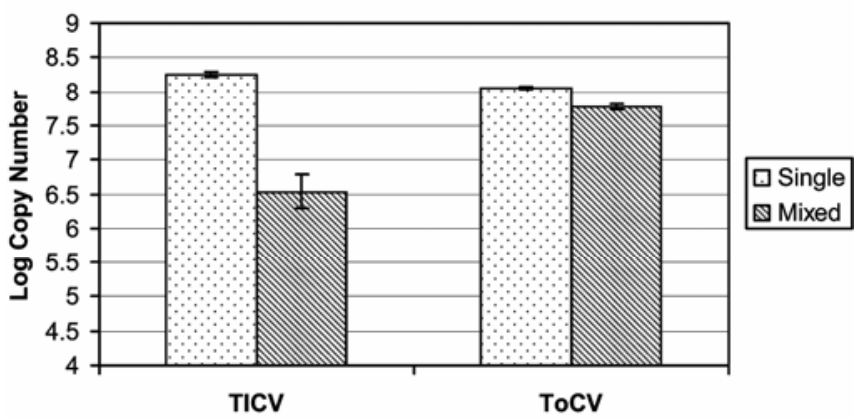

Fig. 2. Accumulation of Tomato infectious chlorosis virus (TICV) and Tomato chlorosis virus (ToCV) in single and mixed infections in Physalis wrightii following transmission by Trialeurodes vaporariorum and T. abutilonea, respectively. Virus accumulation was measured by quantitative reversetranscription polymerase chain reaction as described in Materials and Methods based on standard curves, comparing cycle threshold with target copy number. Quantification of TICV is given by $Y=-3.378 x+38.161$; slope $=-3.378$, intercept $=38.161$, and $R^{2}=0.999$. Quantification of ToCV is given by $Y=$ $-3.567 x+44.041 ;$ slope $=-3.567$, intercept $=44.041$, and $R^{2}=0.999$.
Virus Accumulation in Nicotiana benthamiana

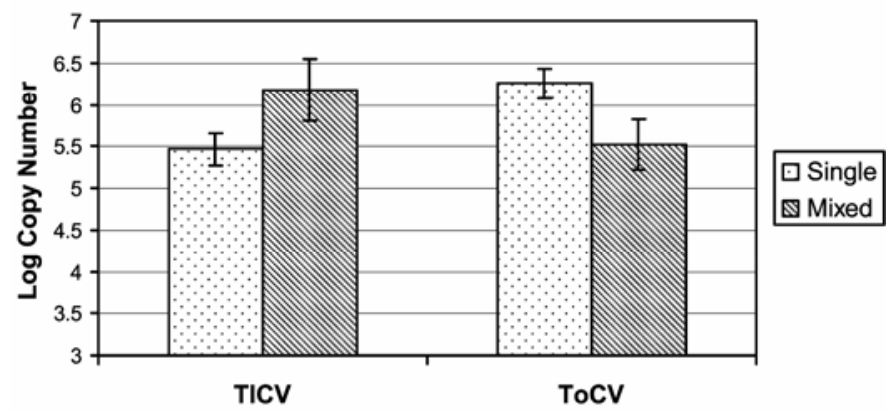

Fig. 3. Accumulation of Tomato infectious chlorosis virus (TICV) and Tomato chlorosis virus (ToCV) in single and mixed infections in Nicotiana benthamiana following transmission by Trialeurodes vaporariorum and $T$. abutilonea, respectively. Virus accumulation was measured by quantitative reverse-transcription polymerase chain reaction as described in Materials and Methods based on a standard curve, comparing cycle threshold with target copy number. Quantification of TICV is given by $Y=-3.378 x+38.161$; slope $=-3.378$, intercept $=38.161$, and $R^{2}=0.999$. Quantification of ToCV is given by $Y=-3.567 x+44.041$; slope $=-3.567$, intercept $=44.041$, and $R^{2}=0.999$. 
(Fig. 3). Accumulation of ToCV was significantly reduced during mixed infection of $N$. benthamiana $(P=0.0016)$, whereas TICV levels actually increased during mixed infection of this host $(P=$ 0.0299).

T. vaporariorum is capable of relatively efficient transmission of ToCV during the first $24 \mathrm{~h}$ following virus acquisition, although still much less efficient than by the other two vectors of this virus under identical conditions. In transmission experiments conducted immediately after a 48-h virus acquisition period on infected $P$. wrightii, TICV and ToCV were transmitted with equal efficiency from single infections (93 and 95\%, respectively) to healthy $P$. wrightii plants by $T$. vaporariorum (Table 1 ). When $P$. wrightii source plants were infected with both viruses, however, ToCV was transmitted much more readily to $P$. wrightii than was TICV (Table 1), even though T. vaporariorum is more efficient at transmission of TICV than ToCV during single infections $(7,23)$. Although TICV transmission from single infections was very efficient, it was difficult to obtain transmission of TICV by its native vector from $P$. wrightii plants co-infected with ToCV. Transmission from mixed infections resulted in only 8 of 53 new plants developing TICV infections, and 6 of these contained mixed infections of TICV and ToCV (Table 1). In contrast, 39 of 53 plants developed infection with ToCV. ToCV was also transmitted well from mixed infections by $T$. abutilonea, its most efficient vector, with a $100 \%$ infection rate from single infections. TICV was not transmitted from single infections by T. abutilonea, which has been demonstrated to be a nonvector of TICV (7). Transmission of ToCV from $P$. wrightii co-infected with TICV and ToCV resulted in $90 \%$ ToCV infection of $P$. wrightii when $T$. abutilonea was the vector (Table 1).

Transmission rates for both viruses by $T$. vaporariorum from singly infected $N$. benthamiana to new $N$. benthamiana plants were statistically identical, although the actual number of ToCVinfected plants resulting from transmissions were slightly higher than for TICV, and fewer plants were involved than in $P$. wrightii experiments (Table 1). In contrast to results with $P$. wrightii, however, $T$. vaporariorum transmission from mixed infections in $N$. benthamiana resulted in higher rates of TICV transmission than ToCV, with 19 of 22 plants becoming infected with TICV following transmission from co-infected source plants compared with 11 of 22 becoming infected with ToCV (Table 1). It should be noted that all plants that became infected with ToCV in experiments involving transmission by $T$. vaporariorum from coinfected plants also became infected with TICV. When T. abutilonea was used as the vector, ToCV was transmitted from single and mixed infections at approximately equal rates; however, in both cases, transmission rates were higher than when ToCV was transmitted to this host by $T$. vaporariorum (Table 1), a less efficient vector of ToCV than T. abutilonea. As in the case of $P$. wrightii, TICV was not transmitted from single infections by $T$. abutilonea.

Although T. abutilonea is believed to be unable to transmit TICV based on extensive previous studies (7), transmission from mixed infections containing approximately equivalent levels of TICV and ToCV resulted in transmission of TICV to individual plants of both $P$. wrightii and $N$. benthamiana by this vector. In all, 1 of $53 P$. wrightii plants and 1 of $45 N$. benthamiana plants tested in transmission experiments became infected with both TICV and ToCV following transmission from mixed infections by T. abutilonea as confirmed using nucleic acid hybridization (Table 1). Two additional experiments were conducted in an attempt to repeat this transmission of TICV by a nonvector from mixed infections in both hosts, but without success; however, the results do suggest the potential for mixed infections to facilitate transmission of a virus by a vector that would not normally be expected to do so. Additional studies on helper-virus-associated transmission of Crinivirus spp. are in progress.

\section{DISCUSSION}

The increased incidence of criniviruses and their whitefly vectors in field and greenhouse production systems across numerous agricultural crops highlights the need for additional efforts toward resistance and management of these viruses. Efforts to elucidate factors contributing to emergence and prevalence of Crinivirus spp. are important for understanding virus epidemiology and developing effective management strategies for virus control. The relationship between Crinivirus spp., their host plants, and their Bemisia and Trialeurodes vectors is central to Crinivirus epidemiology.

The results presented herein suggest that the ability of TICV and ToCV to accumulate in a host is altered during co-infection in a host-specific manner. Although both $P$. wrightii and N. benthamiana are excellent experimental hosts for study and maintenance of both viruses, when the two viruses co-infect the same plant, accumulation patterns for both viruses are altered compared with single infection. In $N$. benthamiana, TICV titers increased during co-infection compared with levels in single infection, whereas ToCV titers decreased (Fig. 3). In P. wrightii, however, titers of both TICV and ToCV decreased during mixed infection compared with single infection, although to different degrees (Fig. 2). Although it may be premature to suggest that these viruses are in direct competition with one another for the ability to replicate, this is clearly a possibility. The results strongly suggest differential adaptation of each virus to each host. These differences may translate into differences in compatibility and, therefore, competitiveness of each virus during co-infection of different hosts, although further studies will be needed to confirm such effects.

Interestingly, this difference in accumulation during mixed infection also translates into differences in transmissibility of each virus during mixed infection. When vector whitefly species fed on $N$. benthamiana or $P$. wrightii plants singly infected with either virus, transmission efficiency was excellent (Table 1). It should be noted that whitefly feeding on $N$. benthamiana is not as aggressive as on $P$. wrightii, but this does not impact Crinivirus transmission. $N$. benthamiana is used routinely in our lab for maintenance of criniviruses as well as serving as a source for Crinivirus transmission studies using a diversity of whitefly vectors (W. M. Wintermantel, unpublished). When plants of either host species containing mixed infections of TICV and ToCV were used as source plants for transmission by vector

TABLE 1. Percent transmission of Tomato infectious chlorosis virus (TICV) and Tomato chlorosis virus (ToCV) from single and mixed virus infections in two hosts by Trialeurodes vaporariorum and T. abutilone $a^{\text {a }}$

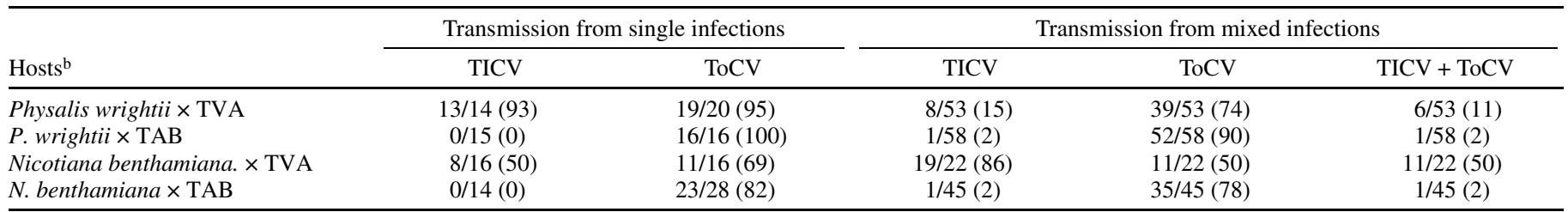

a Number of plants infected/number tested; number in parentheses indicates percent infection.

$\mathrm{b} \mathrm{TVA}=$ Trialeurodes vaporariorum and $\mathrm{TAB}=$ T. abutilonea . 
whiteflies, the rate of transmission as measured by percent infected plants reflected the titer of each virus in the source (Table 1; Figs. 2 and 3).

Recent studies with another Crinivirus sp., Lettuce infectious yellows virus (LIYV), using an in vitro system in which intact LIYV virions were acquired by whiteflies feeding through a membrane, demonstrated that transmission by Bemisia tabaci biotype A was influenced by not only the number of whiteflies used for transmission but also the concentration of virions available for transmission (13). As virions were successively diluted, whitefly transmission decreased. Although this was an in vitro study, these results suggest that LIYV transmission efficiency is likely influenced by virus concentration in the host as well.

Vector transmission efficiency is also a likely determining factor as to whether virus concentration in the host or competition by a co-infecting virus significantly affects virus transmission. Transmission of both TICV and ToCV by $T$. vaporariorum was clearly affected by changes in virus concentration during coinfection in these studies. In contrast, there was little change in the efficiency of ToCV transmission by $T$. abutilonea, a highly efficient ToCV vector, between single and mixed infections in either host (Table 1). Studies have demonstrated that T. abutilonea is not a vector of TICV (7); therefore, co-infection with TICV may have had little impact on transmissibility of ToCV by its most efficient vector.

Inefficient association of TICV with T. abutilonea may explain the low efficiency of TICV transmission by T. abutilonea from mixed infections as well. Results suggest that ToCV may have contributed to transmission of TICV through provision of factors necessary for effective association with $T$. abutilonea. Interestingly, transmission of TICV by T. abutilonea, a whitefly species previously shown to be unable to transmit TICV, occurred with both hosts, indicating that the apparent complementation for transmission may be independent of host factors. Although transmission in association with a helper virus has not been observed previously among whitefly-transmitted viruses, examples have been documented for confirmed and proposed members of the genus Vitivirus (Flexiviridae) (4,18). Although aphid transmitted, vitiviruses are also transmitted semipersistently, as are the whitefly-transmitted Crinivirus spp.

It is clear that numerous factors contribute toward Crinivirus epidemiology and virus emergence and dominance, including whitefly transmission efficiency of a virus, virus titer in a host, and potential interactions resulting from related viruses coinfecting a plant. In the studies described above, the focus was on experimental hosts; however, these studies have relevance to field infections as well. Under field conditions, plants may contain mixed infections involving more than one virus, and it is not uncommon to find mixed infections of different species within the same genus. Co-infection of tomato with TICV and ToCV has been documented in Asia (16). Similarly, Beet pseudo-yellows virus and Strawberry pallidosis-associated virus are frequently found co-infecting strawberry in North America $(17,19)$ and Peru (21). In both cases, the related Crinivirus spp. share common vectors.

Until now, no studies have directly examined the influence of co-infection by two Crinivirus spp. on efficiency of vector transmission. The results presented here have far-reaching implications beyond the family Closteroviridae. The clear differences in virus accumulation between single and mixed infections in two hosts demonstrates the importance of both the host and compatibility of the virus in that host in determining the ability of the virus to accumulate as well as compete for host factors during a mixed infection. Furthermore, the combined effect of these factors clearly has the ability to influence which virus is transmitted most frequently in areas where co-infection is prevalent, and potentially can have a profound impact on emergence and dominance of a virus that is introduced to a new region. Although the frequency of TICV transmission by a whitefly species previously shown through numerous studies to be unable to transmit TICV was low, the occurrence of this event indicates that mixed infections of related viruses may influence virus transmission and suggests potential for viable recombinants to form among Crinivirus spp. infecting the same host. The occurrence of mixed Crinivirus infections is not uncommon in the field and has been documented for a number of virus-vector combinations in several hosts $(16,19,21,26)$.

Once a Crinivirus sp. is introduced to a new area, its unique vector transmissibility and host range determine its ability to establish and recur in the region. As whitefly species and biotypes are inadvertently moved throughout the world on plants and produce, the potential for virus movement increases, as does the potential for co-infection by related viruses that infect similar hosts. Viruses that are transmitted exclusively by a single vector species will not become established in an area where that vector is not present, even if inadvertently introduced. If Crinivirus spp. can complement one another for vector transmission or potentially recombine with one another, the possibility of the emergence of a new virus with different vectoring potential exists. Only through further study of these complex relationships among vectors, hosts, and viruses can we be prepared to manage virus epidemiology in modern agriculture where viruses and vectors are continuously being introduced to new areas through transportation, trade, and environmental factors.

\section{ACKNOWLEDGMENTS}

This project was supported using funds provided through United States Department of Agriculture-Agricultural Research Service project number 5305-22000-010-00D and the California Tomato Commission. We thank I. E. Tzanetakis and R. C. Larsen for critical review of this manuscript.

\section{LITERATURE CITED}

1. ABI. Pages 4-7 in: Creating Standard Curves with Genomic DNA or Plasmid DNA Templates for Use in Quantitative PCR. http://www. appliedbiosystems.com/support/tutorials/pdf/quant_pcr.pdf

2. Abou-Jawdah, Y., El Mohtar, C., Atamian, H., and Sobh, H. 2006. First report of Tomato chlorosis virus in Lebanon. Plant Dis. 90:378.

3. Accotto, G. P., Vaira, A. M., Vecchiati, M., Finetti Sialer, M. M., Gallitelli, D., and Davino, M. 2001. First report of Tomato chlorosis virus in Italy. Plant Dis. 85:1208.

4. Adams, M. J., Antoniw, J. F., Bar-Joseph M., Brunt, A. A., Candresse, T., Foster, G. D., Martelli, G. P., Milne, R. G., Zavriev, S. K., and Fauquet, C. M. 2004. The new plant virus family Flexiviridae and assessment of molecular criteria for species demarcation. Arch. Virol. 149:1045-1060.

5. Dalmon, A., Bouyer, S., Cailly, M., Girard, M., Lecoq, H., Desbiez, C., and Jacquemond, M. 2005. First report of Tomato chlorosis virus and Tomato infectious chlorosis virus in tomato crops in France. Plant Dis. 89:1243.

6. Dovas, C. I., Katis, N. I., and Avgelis, A. D. 2002. Multiplex detection of criniviruses associated with epidemics of a yellowing disease of tomato in Greece. Plant Dis. 86:1345-1349.

7. Duffus, J. E., Liu, H.-Y., and Wisler, G. C. 1996. Tomato infectious chlorosis virus-A new clostero-like virus transmitted by Trialeurodes vaporariorum. Eur. J. Plant Pathol. 102:219-226.

8. Font, M. I., Juarez, M., Martinez, O., and Jorda, C. 2004. Current status and newly discovered natural hosts of Tomato infectious chlorosis virus and Tomato chlorosis virus in Spain. Plant Dis. 88:82.

9. Hanafi, A. 2002. Invasive species: A real challenge to IPM in the Mediterranean region? I. D. Bedford and D. J. Oliver, eds. EWSN Newsl. No. 13.

10. Li, R. H., Wisler, G. C., Liu, H.-Y., and Duffus, J. E. 1998. Comparison of diagnostic techniques for detecting tomato infectious chlorosis virus. Plant Dis. 82:84-88.

11. Louro, D., Accotto, G. P., and Vaira, A. M. 2000. Occurrence and diagnosis of Tomato chlorosis virus in Portugal. Eur. J. Plant Pathol. 106:589-592.

12. Navas-Castillo, J., Camero, R., Bueno, M., and Moriones, E. 2000. Severe yellowing outbreaks in tomato in Spain associated with infections of Tomato chlorosis virus. Plant Dis. 84:835-837.

13. Ng, J. C. K., Tian, T., and Falk, B. W. 2004. Quantitative parameters 
determining whitefly (Bemisia tabaci) transmission of Lettuce infectious yellows virus and an engineered defective RNA. J. Gen. Virol. 85:26972707.

14. Pfaffl, M. W., and Hageleit, M.. 2001. Validities of mRNA quantification using recombinant RNA and recombinant DNA external calibration curves in real-time RT-PCR. Biotechnol. Lett. 23:275-282.

15. Segev, L., Wintermantel, W. M., Polston, J. E., and Lapidot, M. 2004. First report of Tomato chlorosis virus in Israel. Plant Dis. 88:1160.

16. Tsai, W. S., Shih, S. L., Green, S. K., Hanson, P., and Liu, H. Y. 2004. First report of the occurrence of Tomato chlorosis virus and Tomato infectious chlorosis virus in Taiwan. Plant Dis. 88:311.

17. Tzanetakis, I. E., Halgren, A. B., Keller, K. E., Hokanson, S. C., Maas, J. L., McCarthy, P. L., and Martin, R. R. 2004. Identification and detection of a virus associated with strawberry pallidosis disease. Plant Dis. 88:383390.

18. Tzanetakis, I. E., Postman, J. D., and Martin, R. R. 2007. Identification, detection and transmission of a new Vitivirus from Mentha. Arch. Virol. 152:2027-2033.

19. Tzanetakis, I. E., Wintermantel, W. M., Cortez, A. A., Barnes, J. E., Barrett, S. M., Bolda, M. P., and Martin, R. R. 2006. Epidemiology of Strawberry pallidosis-associated virus and occurrence of pallidosis disease in North America. Plant Dis. 90:1343-1346.

20. Wintermantel, W. M. 2004. Emergence of greenhouse whitefly (Trialeurodes vaporariorum) transmitted criniviruses as threats to vegetable and fruit production in North America. APSnet feature. Published online by The American Phytopathological Society, St. Paul, MN.
21. Wintermantel, W. M., Fuentes, S., Chuquillanqui, C., and Salazar, L. F. 2006. First report of Beet pseudo-yellows virus and Strawberry pallidosis associated virus in strawberry in Peru. Plant Dis. 90:1457.

22. Wintermantel, W. M., Polston, J. E., Escudero, J., and Paoli, E. R. 2001. First report of Tomato chlorosis virus in Puerto Rico. Plant Dis. 85:228.

23. Wintermantel, W. M., and Wisler, G. C. 2006. Vector specificity, host range, and genetic diversity of Tomato chlorosis virus. Plant Dis. 90:814819.

24. Wintermantel, W. M., Wisler, G. C., Anchieta, A. G., Liu, H.-Y., Karasev, A. V., and Tzanetakis, I. E. 2005. The complete nucleotide sequence and genome organization of Tomato chlorosis virus. Arch. Virol. 150:22872298.

25. Wisler, G. C., and Duffus, J. E. 2001. Transmission properties of whitefly-borne criniviruses and their impact on virus epidemiology. Pages 293-308 in: Virus-Insect-Plant Interactions. K. F. Harris, O. P. Smith, and J. E. Duffus, eds. Academic Press, San Diego, CA.

26. Wisler, G. C., Duffus, J. E., Liu, H.-Y., and Li, R. H. 1998. Ecology and epidemiology of whitefly-transmitted closteroviruses. Plant Dis. 82:270280 .

27. Wisler, G. C., Li, R. H., Liu, H.-Y., Lowry, D., and Duffus, J. E. 1998. Tomato chlorosis virus: A new whitefly-transmitted, phloem-limited bipartite closterovirus of tomato. Phytopathology 88:402-409.

28. Wisler, G. C., Liu, H. J.-Y., Klaassen, V. A., Duffus, J. E., and Falk, B. W. 1996. Tomato infectious chlorosis virus has a bipartite genome and induces phloem-limited inclusions characteristic of the closteroviruses. Phytopathology 86:622-626. 